\title{
INVESTIGATING THE EFFECTS OF CUSTOMER RELATIONSHIP MANAGEMENT AND SUPPLIER RELATIONSHIP MANAGEMENT ON NEW PRODUCT DEVELOPMENT
}

\author{
Salman Nazari-Shirkouhi, Abbas Keramati, Kamran Rezaie
}

Original scientific paper

Most organizations today are increasingly influenced by competition and global markets due to advancements in information technology and economic globalization. Developments in information technology and increasing competitiveness are led to shorter product life cycles, increase rate of product obsolescence, growth of high-quality products and introduction of new products into the market. These factors have stimulated organizations to follow NPD goals such as reducing product development time, lower product cost with higher quality, shorter time-to-market, and improving delivery performance to achieve profitability and keep their market position. Customer and supplier involvement during the NPD processes has attracted widespread attention in the literature. SRM and CRM can improve the performance of New Product Development (NPD) as well as facilitate the role of customers and suppliers in NPD in the path towards a high-performance organization; therefore, it is necessary for organizations to gain a correct understanding of the roles of CRM and SRM in NPD processes and to draw on such a knowledge to enhance the performance of the new product. Given the advantages of applying CRM and SRM within organizations, this paper tries to explore the different roles of CRM and SRM which play in NPD process.

Keywords: customer; customer relationship management; new product development; supplier; supplier relationship management

Istraživanja učinaka upravljanja odnosa s kupcima i upravljanja odnosa s dobavljačima na razvoj novog proizvoda

Izvorni znanstveni članak

$\mathrm{Na}$ većinu poduzeća danas sve više utječu konkurencija i globalna tržišta zbog usavršavanja informatičke tehnologije i ekonomske globalizacije. Razvoj informatičke tehnologije i sve veća stručnost dovode do kraćeg vijeka trajanja proizvoda, bržeg zastarijevanja proizvoda, povećanja broja visokokvalitetnih proizvoda i stavljanja novih proizvoda na tržište. Ove su činjenice potaknule proizvodne organizacije da slijede ciljeve razvoja novih proizvoda (NPD) kao što su smanjenje vremena razvoja proizvoda, niža cijena proizvodnje uz bolju kvalitetu, skraćivanje vremena plasmana proizvoda na tržište, bolji uvjeti isporuke u svrhu profitabilnosti i opstanka na tržištu. Uključivanje kupaca i dobavljača u proces NPD postalo je vrlo popularna tema u literaturi. SRM i NPD mogu unaprijediti postupak razvoja novog proizvoda (NPD) i olakšati ulogu kupaca i dobavljača u NPD u cilju razvoja visoko produktivne organizacije; stoga organizacije trebaju dobro razumjeti uloge CRM i SRM u postupku razvoja novog proizvoda i stečeno znanje iskoristiti za unapređenje novih proizvoda. Zbog prednosti primjene CRM i SRM u proizvodnim organizacijama, u radu se istražuju različite uloge CRM i SRM u postupku razvoja novog proizvoda.

Ključne riječi: dobavljač; kupac; razvoj novog proizvoda; upravljanje odnosa s dobavljačima; upravljanje odnosa s kupcima

\section{Introduction}

With many companies facing highly unpredictable market conditions and with huge advancements in technology, organizations are required to push for the enhancement of their capabilities in New Product Development (NPD) in their attempts to satisfy market demands and customer preferences. As long as production of new products can lead to profit growth and a higher sale rate, NPD is one of the strategic activities in many organizations. In fact new products could be considered as one of the key factors in success of organizations in the market. However, whereas the role of NPD and innovation strategies in building of competitive advantage and improving financial performance have been studied extensively in the literature, new products have failed to achieve the considered goals in many cases. Numerous variables have been mentioned as factors leading to a successful NPD in literature. Customer and supplier involvement during the NPD processes has attracted considerable attention in the literature $[1,2]$.One effective strategy for reducing the failure rate of new products, on the one hand, and increasing their success rate on the other is to align the new product development process with market requirements [3, 4]. Positive effects of customer orientation and applying the customers' information on new product development have been frequently documented in the literature. Previous studies on supply chain and operation management suggest different methods for organizations to improve their competitive advantage through cooperation and coordination with suppliers. For instance, according to Rauniar et al. [5], knowledge sharing among customers, suppliers, and the development team members can decrease the defects of NPD project and improve time, cost, and customer satisfaction of product development. Many researchers have separately conducted works related to supplier involvement or customer involvement in NPD, but our knowledge there has been a limited focus on studying the effects of the simultaneous involvement of both suppliers and customers in NPD (e.g. See. Sun et al. [6], Chien \& Chen [7]).

Additionally, although relevant studies have helped organizations gain insight into factors influencing on supplier involvement and customer involvement in NPD, these studies have only begun to introduce an integrated framework for applying supplier and customer knowledge in order to create successful and sustainable product development. Therefore, the aim of this paper is to introduce and develop such a framework for NPD by applying both supplier relationship management (SRM) and customer relationship management (CRM) concepts simultaneously. Not only CRM is able to maintain organization's existing customers, it is also effective in encouraging customers to offer more suggestions and implications to enhance the quality of products and services [8]. In fact CRM would help organizations achieve relevant knowledge about customers' preferences. 
CRM has also proved to be a highly effective and efficient tool to develop innovation capabilities and create competitive advantage within an organization $[8 \div 10]$.

CRM involves all activities that organizations establish long-term, continuous, profitable and mutually beneficial relationships with customers. Reinartz et al.[11] consider CRM as a process for customer interaction management to build, develop, and maintenance longterm and beneficial relationships with customers. In summary, CRM has capabilities which allow customerrelated information to be systematically used in NPD process in ways that could improve new product performance.

SRM, on the other hand, is the discipline of managing preferred suppliers and finding ways to reduce costs, supply goods and materials repeatability, share the experiences of organizations-suppliers and extract relevant advantages from the relationships between organizations and suppliers [12]. SRM is focused on maximizing the value of the relationship between organizations and suppliers by providing an integrated set of management tools in order to interact organization with its suppliers.

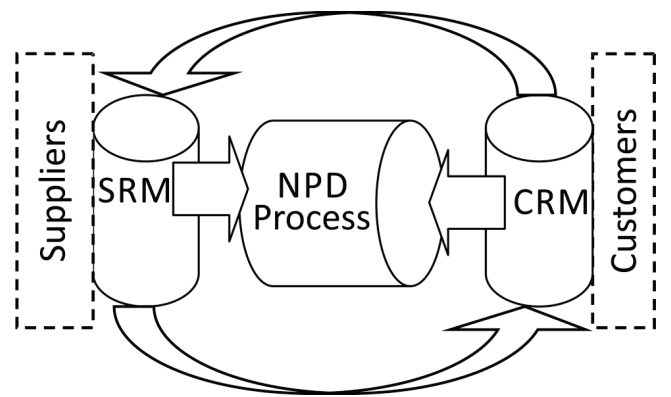

Figure 1CRM, SRM, and NPD

The strategic objective of SRM is to collaborate with suppliers so that a company can produce new and efficient products [13]. SRM is a category of supply chain which can deliver many competitive advantage contributions through leveraging the business processes in supply chain as well as facilitating the acceleration of product development cycles and introduction of new products into the market. Such mechanisms are able to drive competitive advantage by reductions in the costs of parts and materials, increasing flexibility and responsiveness to changes in the customers' demands, and accelerating cycle time which could in turn enhance customer satisfaction and increase market share. Accordingly, the need for an integrated system which can take into account the roles of CRM and SRM in new product development is increasingly felt in organizations. As depicted in Fig. 1, the relationships between suppliers, customers and new product development is established in such a system. Given the relationships between CRM, SRM and new product development, it is expected that advantages such as cost-effectiveness, higher flexibility and responsiveness to changes in customers' demands, lower cycle time and enhanced quality of products would bring about the customers' satisfaction and finally the improvement of the organizational performance.

\section{Key factors in the success of new product development}

Notwithstanding the focus on the importance of the NPD process in organizations, high rates of failure, sometimes as high as $40 \%$, has been reported in the literature for new products [14]. Success rate has been reported to be less than $60 \%$ in some countries; for example, the rate has been reported at 54,3 and 59,8 \% for England and Japan, respectively [15], at $59 \%$ for United States [16] and at $49 \%$ for Spain [17]. A study by Tennant \& Roberts [18] suggested only $23 \%$ of the 200 firms surveyed delivered their products on time. Several factors have been suggested in the literature influencing the success of new products. For example, Lettice et al. [19] compiled 200 factors and categorized them into six dimensions, i.e. stakeholders' contributions, invention, operating context, exploitation, reuse and the performance of stakeholders in New product Development, which is in turn divided into customers, suppliers, senior management, production/delivery and marketing; Operating context includes human resources, technological infrastructure, organizational structure and competitive context; Reuse of assets includes knowledgerelated assets, processes, organization and reuse library use. Invention includes invention portfolio, invention process, and invention organization and tools; finally, exploitation includes commercialization criteria. Generally, key factors extracted from literature could be classified as process, employee-oriented, strategic, management, and information technology.

\section{Customer involvement in new product development}

Customer involvement in the innovation process is one of the most important and valuable resources available to organizations. Using these opportunities, organizations can enhance innovation capabilities and reduce risks related to producing innovative products [20]. Customer involvement in the NPD process can lead to identifying customers' needs, gathering relevant customer information and translating customers' needs into new products. Moreover, use of customers' knowledge can increase new product advantage for organizations and enable them to discover opportunities for innovation and idea generation. In general, successful companies have been found to spend a lot of time on gathering and analysing customer-related information, studying market, identifying lead users and cooperating with them, as well as on learning activities and other methods for absorbing knowledge [21]. With the emergence of information systems, many organizations have used the technologies to apply and share knowledge with their customers or participate in activities related to value creation (e.g. new product development). Examples of such organizations include Cisco, Bang \& Olufsen, 3Com, Microsoft, Fiat and Compaq. According to Nambisan [22], customers can play three roles (resource, co-creator, user) in NPD. Customer as resource is related to a source of innovation and idea generation, customer as co-creator is related to customer participation in product design and development phase, and customer as user is related to customer involvement in product testing and 
support. Accordingly, organizations' close relationships with their customers and customers' participation through NPD process could be considered as key success factors in new product development.

\section{Supplier involvement in new product development}

According to reports by LLC performance evaluation [23], most organizations endeavour to increase participation rate by external parties outside organizations in new product development. Various definitions of supplier involvement in product development have been used in previous studies. Wagner [24] defines supplier integration in new product development as "the combination of internal resources of the buying firm with the resources and capabilities of selected key suppliers through the meshing of intercompany business processes to achieve a competitive advantage". Van Echteltetal [25] provides the following definition: "the resources (capabilities, investments, information, knowledge, ideas) that suppliers provide, the tasks they carry out and the responsibilities they assume regarding the development of a part, process or service for the benefit of a buyer's current or future product development projects."

Most organizations depend on their supplier involvement in new product development in order to increase their innovative capabilities and their products' quality [26]. Many researchers have mentioned the advantages of supplier involvement in new product development $[26 \div 30]$. These advantages include new product time to market, product quality, development cost, product cost, cycle time and etc. In general, supplier involvement in new product development has potential advantages such as helping organizations achieve new competencies, risk sharing, quick entry into new markets. On the other hand, some other studies in the literature indicate the negative impacts of such integration on performance. It is claimed that the involvement of suppliers in product development leads to a higher cost of production as well as a lower performance and a more lengthy development time [31]. For example, having studied 106 companies involved in information and communication technology industries, Littler et al. [32] concluded product development was more costly and less efficient because supplier involvement imposes the particular complexities upon project management. Similarly, Hartley et al. [33] concluded that relationships with suppliers do not influence the NPD time performance. Key factors from literature are supplier selection process with consideration of culture of suppliers and their capabilities, timing and extent of supplier integration, information sharing with suppliers etc.

\section{A review of the models on variables related to customer involvement and NPD}

Several theoretical and practical studies have examined the relationship between factors related to customers and NPD performance. This section reviews studies that have examined this relationship. Tab.1 shows related studies.

Table 1 A summary of studies related to customer involvement in NPD and its impact on performance

\begin{tabular}{|c|c|c|c|c|}
\hline Reference & $\begin{array}{l}\text { Independent } \\
\text { variables }\end{array}$ & Dependent variables & $\begin{array}{c}\text { Performance } \\
\text { variables }\end{array}$ & Results \\
\hline 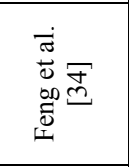 & Time to market & $\begin{array}{l}\text { Customer focus, } \\
\text { customer involvement, } \\
\text { communication with } \\
\text { customers }\end{array}$ & Time to market & $\begin{array}{l}\text { Customer focus, customer involvement, and communication with } \\
\text { customers are significantly and negatively related to time-to-market. } \\
\text { Negative direct of moderating effects of it implementation on } \\
\text { relationship between customer involvement/ communication with } \\
\text { customers and time to market. }\end{array}$ \\
\hline $\begin{array}{l}\Xi \\
\text { Е } \\
\text { ¿ } \\
\Xi \\
\Xi\end{array}$ & $\begin{array}{l}\text { Information sharing, } \\
\text { Customer } \\
\text { involvement, Long- } \\
\text { term partnership, } \\
\text { Joint problem- } \\
\text { solving, Technology- } \\
\text { based CRM }\end{array}$ & $\begin{array}{l}\text { product innovation, } \\
\text { process innovation, } \\
\text { administrative } \\
\text { innovation, marketing } \\
\text { innovation, service } \\
\text { innovation }\end{array}$ & - & $\begin{array}{l}\text { Significant relationship between information sharing and: } \\
\text { product/process/service innovation. Significant relationship between } \\
\text { customer involvement and: product/marketing/ service innovation. } \\
\text { Significant relationship between technology-based CRM and } \\
\text { product/process/administrative/marketing/service innovation. }\end{array}$ \\
\hline 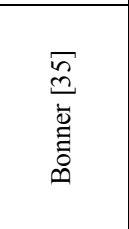 & $\begin{array}{l}\text { Customer } \\
\text { interactivity }\end{array}$ & $\begin{array}{c}\text { Customer information } \\
\text { quality, New product } \\
\text { performance }\end{array}$ & $\begin{array}{l}\text { Product quality, } \\
\text { Product features, } \\
\text { Technical product } \\
\text { performance, } \\
\text { Meeting customer } \\
\text { needs }\end{array}$ & $\begin{array}{l}\text { Significant relationship between customer interactivity and customer } \\
\text { information quality. Significant relationship between customer } \\
\text { information quality and new product performance. Positive direct of } \\
\text { moderating effects of product newness on relationship between } \\
\text { customer interactivity and customer information quality. Positive } \\
\text { direct of moderating effects of product embeddedness on relationship } \\
\text { between customer interactivity and customer information quality. }\end{array}$ \\
\hline 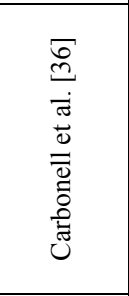 & $\begin{array}{l}\text { technological } \\
\text { uncertainty } \\
\text { (Technological } \\
\text { novelty, } \\
\text { Technological } \\
\text { turbulence) }\end{array}$ & $\begin{array}{l}\text { Customer } \\
\text { Involvement, } \\
\text { Innovation Speed, } \\
\text { Technical Quality, } \\
\text { Competitive } \\
\text { Superiority, Sales } \\
\text { performance }\end{array}$ & $\begin{array}{c}\text { Operational } \\
\text { outcomes } \\
\text { (Innovation } \\
\text { speed Technical } \\
\text { Quality). Market } \\
\text { outcomes } \\
\text { (Competitive } \\
\text { Superiority, Sales } \\
\text { performance) }\end{array}$ & $\begin{array}{l}\text { Significant relationship between customer involvement and: } \\
\text { innovation speed, technical quality. Significant relationship between } \\
\text { technological. } \\
\text { Novelty and customer involvement; significant relationship between } \\
\text { technological turbulence and customer involvement. }\end{array}$ \\
\hline 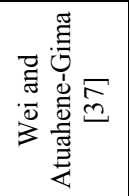 & Market orientation & $\begin{array}{l}\text { New Product } \\
\text { Performance }\end{array}$ & $\begin{array}{l}\text { percentage of sales, } \\
\text { percentage of } \\
\text { profits, percentage } \\
\text { of market share) }\end{array}$ & $\begin{array}{l}\text { Significant relationship between market orientation and NPD } \\
\text { performance. Negative direct of moderating effects of risk-taking } \\
\text { rewards on relationship between market orientation and NPD } \\
\text { performance. Positive direct of moderating effects of Long-term } \\
\text { rewards on relationship between market orientation and NPD } \\
\text { performance. }\end{array}$ \\
\hline
\end{tabular}




\begin{tabular}{|c|c|c|c|c|}
\hline 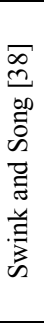 & \begin{tabular}{|c|} 
Manufacturing- \\
marketing integration \\
during four stages of \\
the NPD project \\
(Business/market \\
analysis, technical \\
development, \\
product testing, \\
product \\
commercialization) \\
\end{tabular} & $\begin{array}{l}\text { NPD project length, } \\
\text { product competitive } \\
\text { advantages, NPD } \\
\text { project ROI }\end{array}$ & $\begin{array}{l}\text { return on } \\
\text { investment during } \\
\text { the first } 12 \text { months }\end{array}$ & $\begin{array}{l}\text { Significant relationship between Manufacturing-marketing integration } \\
\text { and product competitive advantages. Significant relationship between } \\
\text { Manufacturing-marketing integration and: Business/market analysis } \\
\text { length and product commercialization length. Significant relationship } \\
\text { between competitive advantages and NPD project ROI. }\end{array}$ \\
\hline 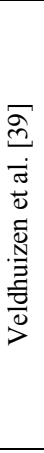 & $\begin{array}{l}\text { Product newness } \\
\text { to the market, project } \\
\text { urgency (project } \\
\text { priority, time } \\
\text { pressure), company } \\
\text { characteristics (R\&D } \\
\text { Dominance, } \\
\text { interdepartmental } \\
\text { conflict, flexibility to } \\
\text { new products) }\end{array}$ & $\begin{array}{l}\text { use of market } \\
\text { information during } \\
\text { NPD projects } \\
\text { (predevelopment/devel } \\
\text { opment/commercializa } \\
\text { tion),Dissemination of } \\
\text { market information, } \\
\text { Acquisition of } \\
\text { customer information, } \\
\text { Acquisition of } \\
\text { environmental } \\
\text { information, Product } \\
\text { advantage, NPD } \\
\text { success }\end{array}$ & $\begin{array}{c}\text { NPD success } \\
\text { (Market/financial } \\
\text { success, Time/cost } \\
\text { efficiency) } \\
\text { Product advantage } \\
\text { (quality, innovative, } \\
\text { superior to } \\
\text { competing } \\
\text { products) }\end{array}$ & $\begin{array}{l}\text { Significant relationship between Product advantage and NPD success. } \\
\text { Significant relationship between use of market information during } \\
\text { commercialization stage and Product advantage. Significant } \\
\text { relationship between Acquisition of customer information and: use in } \\
\text { development, Product advantage. Significant relationship between use } \\
\text { of market information during predevelopment stage and } \\
\text { Market/financial success. Significant relationship between } \\
\text { dissemination of market information and use of market information } \\
\text { during NPD projects (predevelopment/commercialization), significant } \\
\text { relationship between Acquisition of environmental information and } \\
\text { use in predevelopment. Significant relationship between Product } \\
\text { newness to the market and product advantage. Significant relationship } \\
\text { between project priority and: Acquisition of environmental } \\
\text { information, dissemination of market information. }\end{array}$ \\
\hline 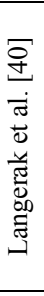 & $\begin{array}{l}\text { Market orientation } \\
\text { (customer } \\
\text { orientation, } \\
\text { competitor } \\
\text { orientation, and } \\
\text { interfunctional } \\
\text { coordination) }\end{array}$ & $\begin{array}{l}\text { Product advantage, } \\
\text { Market testing, } \\
\text { Launch budgeting, } \\
\text { Launch strategy, } \\
\text { Launch tactics, New } \\
\text { product } \\
\text { Performance, } \\
\text { organizational } \\
\text { performance. }\end{array}$ & $\begin{array}{l}\text { New Product } \\
\text { Performance; } \\
\text { Organizational }\end{array}$ & $\begin{array}{l}\text { Significant relationship between Market orientation and: Product } \\
\text { advantage, Launch tactics, Organizational performance. Significant } \\
\text { relationship between Product advantage and New product } \\
\text { Performance. Significant relationship between Launch tactics and } \\
\text { New product performance. }\end{array}$ \\
\hline
\end{tabular}

\section{A review of the models on variables related to supplier involvement and NPD}

Several researches have attempted both theoretically and practically to study the relationships between factors related to suppliers and NPD performance. This section will review studies having explored such relationships. Tab. 2 provides a summary of these studies.

\section{A review of existing models considering both customer and supplier involvement in NPD}

Few studies have considered the relationship between both customer and supplier involvement in NPD process and NPD performance either theoretically or practically. Tab. 3 summarizes the studies having considered this relationship. Models include Koufteros et al. [47], Lau et al. [48], Sun et al. [6], Lau et al. [48] and Chien \& Chen [7].

Table 2 A summary of studies related to supplier involvement in NPD and its impact on performance

\begin{tabular}{|c|c|c|c|c|}
\hline Reference & $\begin{array}{l}\text { Independent } \\
\text { variables }\end{array}$ & Dependent variables & $\begin{array}{l}\text { Performance } \\
\text { variables }\end{array}$ & Results \\
\hline 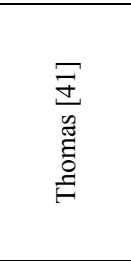 & $\begin{array}{l}\text { Computer mediated } \\
\text { communication } \\
\text { channels (Face-to- } \\
\text { Face, Video } \\
\text { Conferencing, } \\
\text { Email, Web-based } \\
\text { Tools) }\end{array}$ & $\begin{array}{l}\text { Knowledge } \\
\text { Exchange between } \\
\text { buyer and supplier } \\
\text { firms, Buyer NPD } \\
\text { Performance } \\
\text { (Effectiveness, } \\
\text { Efficiency), Buyer } \\
\text { Market Performance }\end{array}$ & $\begin{array}{c}\text { Effectiveness, } \\
\text { Efficiency, Buyer } \\
\text { Market } \\
\text { Performance }\end{array}$ & $\begin{array}{l}\text { Significant and positive relationship between knowledge exchange } \\
\text { between buyer and supplier firms and both NPD effectiveness and } \\
\text { NPD efficiency. Full mediation with face-to-face communication and } \\
\text { partial mediation for email and web-based communication via } \\
\text { knowledge exchange with regard to NPD effectiveness. Full } \\
\text { mediation with email communication and partial mediation with face- } \\
\text { to-face communication via knowledge exchange with regard to NPD } \\
\text { efficiency }\end{array}$ \\
\hline 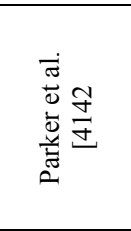 & $\begin{array}{l}\text { Technological } \\
\text { Newness, } \\
\text { Buyer/Supplier } \\
\text { Relationship, } \\
\text { Strategic } \\
\text { Importance of the } \\
\text { Supplied Item }\end{array}$ & $\begin{array}{l}\text { Timing of Integration, } \\
\text { Extent of Integration, } \\
\text { Project Performance }\end{array}$ & $\begin{array}{c}\text { Project } \\
\text { Performance }\end{array}$ & $\begin{array}{l}\text { Significant relationship between technological newness and timing of } \\
\text { integration. Significant relationship between buyer/supplier } \\
\text { relationship and extent of integration. Significant relationship } \\
\text { between strategic importance of the supplied item and extent of } \\
\text { integration. Significant relationship between timing of integration and } \\
\text { extent of integration. Significant relationship between extent of } \\
\text { integration and project performance. }\end{array}$ \\
\hline 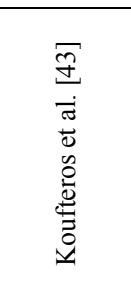 & $\begin{array}{l}\text { Embeddedness with } \\
\text { suppliers }\end{array}$ & $\begin{array}{l}\text { Supplier selection } \\
\text { based on } \\
\text { product development } \\
\text { capabilities, Supply } \\
\text { base rationalization, } \\
\text { Black-box integration, } \\
\text { Gray-box integration, } \\
\text { product innovation, } \\
\text { external quality }\end{array}$ & external quality & $\begin{array}{l}\text { Significant relationship between Embeddedness with suppliers and: } \\
\text { Supplier selection based on product development capabilities, Supply } \\
\text { base rationalization. Significant relationship between Supply base } \\
\text { rationalization and Gray-box integration. Significant relationship } \\
\text { between Supplier selection based on product development capabilities } \\
\text { and: Black-box integration, Gray-box integration. Significant } \\
\text { relationship between Gray-box integration and product innovation. } \\
\text { Significant relationship between product innovation and external } \\
\text { quality. }\end{array}$ \\
\hline
\end{tabular}




\begin{tabular}{|c|c|c|c|c|}
\hline 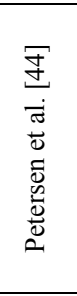 & $\begin{array}{c}\text { Detailed supplier } \\
\text { assessment, technical } \\
\text { assessment, business } \\
\text { assessment }\end{array}$ & $\begin{array}{l}\text { Project team } \\
\text { effectiveness, financial } \\
\text { performance, design } \\
\text { performance }\end{array}$ & $\begin{array}{c}\text { financial } \\
\text { performance, } \\
\text { design performance }\end{array}$ & $\begin{array}{l}\text { Significant relationship between detailed supplier assessment and } \\
\text { project team effectiveness. Significant relationship between technical } \\
\text { assessment and project team effectiveness. Significant relationship } \\
\text { between project team effectiveness and: financial performance, design } \\
\text { performance. Positive direct of moderating effects of early supplier } \\
\text { integration on relationship between project team effectiveness and } \\
\text { design performance. Positive direct of moderating effects of level of } \\
\text { responsibility (black-box) on relationship between technical } \\
\text { assessment and project team effectiveness. }\end{array}$ \\
\hline 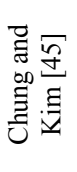 & $\begin{array}{l}\text { Level of supplier } \\
\text { involvement in new } \\
\text { product development } \\
\text { (SINPD) }\end{array}$ & $\begin{array}{l}\text { suppliers' level of } \\
\text { innovation, suppliers' } \\
\text { product quality, } \\
\text { suppliers' financial } \\
\text { performance }\end{array}$ & $\begin{array}{c}\text { financial } \\
\text { performance, } \\
\text { quality, innovation }\end{array}$ & $\begin{array}{l}\text { Significant relationship between higher level of SINPD and: } \\
\text { suppliers' level of innovation, suppliers' financial performance, } \\
\text { suppliers' product quality in the electronics industry. Significant } \\
\text { relationship between higher level of supplier involvement in design } \\
\text { stage and: suppliers' innovation and cash-flow rate. }\end{array}$ \\
\hline 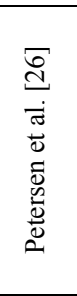 & $\begin{array}{c}\text { Customer } \\
\text { Knowledge of } \\
\text { Supplier, Technology } \\
\text { Uncertainty }\end{array}$ & $\begin{array}{l}\text { technology and cost } \\
\text { information sharing, } \\
\text { supplier involvement } \\
\text { in decision-making, } \\
\text { project outcomes }\end{array}$ & $\begin{array}{l}\text { project outcomes } \\
\text { (Degree of overall } \\
\text { satisfaction with } \\
\text { results, Overall } \\
\text { degree of } \\
\text { achievement to } \\
\text { goals }\end{array}$ & $\begin{array}{l}\text { Significant relationship between customer knowledge of supplier and: } \\
\text { supplier involvement in decision-making, technology and cost } \\
\text { information sharing. Significant relationship between technology and } \\
\text { cost information sharing and: involvement in decision-making, } \\
\text { project outcomes. Significant relationship between supplier } \\
\text { involvement in decision-making and project outcomes. Significant } \\
\text { relationship between technology uncertainty and technology and cost } \\
\text { information sharing. Negative significant relationship between } \\
\text { technology uncertainty and technology and cost information sharing }\end{array}$ \\
\hline 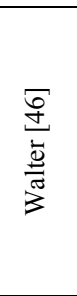 & $\begin{array}{c}\text { relationship } \\
\text { advancement by a } \\
\text { relationship promoter } \\
\text { of the customer }\end{array}$ & $\begin{array}{l}\text { Supplier-specific } \\
\text { adaptations of the } \\
\text { customer, Supplier } \\
\text { trust, Supplier } \\
\text { commitment, supplier } \\
\text { involvement in } \\
\text { customer NPD }\end{array}$ & - & $\begin{array}{l}\text { Significant relationship between Supplier commitment and supplier } \\
\text { involvement in NPD. Significant relationship between Supplier trust } \\
\text { and supplier involvement in NPD. Significant relationship between } \\
\text { relationship advancement by a relationship promoter of the customer } \\
\text { and: supplier involvement in NPD, Supplier trust, Supplier } \\
\text { commitment. Significant relationship between Supplier trust and } \\
\text { Supplier commitment, Supplier-specific adaptations of the customer. } \\
\text { Significant relationship between Supplier-specific adaptations of the } \\
\text { customer and: Supplier trust, Supplier commitment. }\end{array}$ \\
\hline
\end{tabular}

Table 3 A summary of studies related to both customer and supplier involvement in NPD and their impact on performance

\begin{tabular}{|c|c|c|c|c|}
\hline Reference & $\begin{array}{l}\text { Independent } \\
\text { variables }\end{array}$ & Dependent variables & $\begin{array}{l}\text { Performance } \\
\text { variables }\end{array}$ & Results \\
\hline 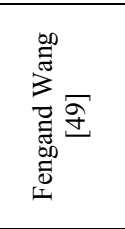 & $\begin{array}{c}\text { Customer } \\
\text { Involvement, Internal } \\
\text { Involvement, } \\
\text { Supplier } \\
\text { Involvement }\end{array}$ & $\begin{array}{l}\text { NPD Cost, Market } \\
\text { Performance, NPD } \\
\text { Speed }\end{array}$ & $\begin{array}{l}\text { NPD Cost, Market } \\
\text { Performance, NPD } \\
\quad \text { Speed }\end{array}$ & $\begin{array}{l}\text { Significant relationship between internal involvement and both } \\
\text { customer involvement and supplier involvement. Significant } \\
\text { relationship between customer involvement and both NPD cost and } \\
\text { NPD speed. Significant relationship between supplier involvement } \\
\text { and NPD cost/ market performance/NPD speed. Significant } \\
\text { relationship between NPD cost and market performance. Significant } \\
\text { relationship between NPD speed and market performance. }\end{array}$ \\
\hline $\begin{array}{l}\stackrel{\infty}{+\infty} \\
\underset{\Xi}{\Xi}\end{array}$ & $\begin{array}{c}\text { Product } \\
\text { Innovativeness, } \\
\text { Product Modularity, } \\
\text { Internal coordination }\end{array}$ & $\begin{array}{l}\text { Supplier and customer } \\
\text { Involvement (SCI), } \\
\text { New product } \\
\text { performance }\end{array}$ & $\begin{array}{l}\text { New product } \\
\text { Performance } \\
\text { (achieve to sales } \\
\text { goal, profit goal, } \\
\text { profitability, } \\
\text { customer } \\
\text { satisfaction) } \\
\end{array}$ & $\begin{array}{l}\text { Significant relationship between product innovativeness and: new } \\
\text { product performance. Significant relationship between product } \\
\text { innovativeness, product modularity, internal coordination and sci. } \\
\text { Significant relationship between SCI and new product performance. }\end{array}$ \\
\hline$\underset{\Xi}{\stackrel{\pi}{\pi}}$ & $\begin{array}{l}\text { Supplier } \\
\text { Involvement, } \\
\text { Customer } \\
\text { involvement }\end{array}$ & $\begin{array}{l}\text { Quality and } \\
\text { Reliability, Time to } \\
\text { Market, } \\
\text { Innovativeness }\end{array}$ & $\begin{array}{l}\text { Quality and } \\
\text { Reliability, Time to } \\
\text { Market, } \\
\text { Innovativeness }\end{array}$ & $\begin{array}{l}\text { Significant relationship between Supplier Involvement and: Quality } \\
\text { and Reliability, Time to Market, Innovativeness. Significant } \\
\text { relationship between Customer involvement Quality and Reliability. }\end{array}$ \\
\hline 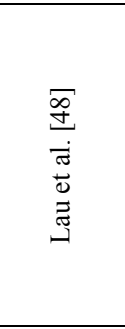 & $\begin{array}{l}\text { Information sharing } \\
\text { with supplier/ } \\
\text { customer, Product } \\
\text { co-development with } \\
\text { supplier/ customer }\end{array}$ & $\begin{array}{l}\text { Product Innovation, } \\
\text { Product performance }\end{array}$ & $\begin{array}{l}\text { Product Innovation, } \\
\text { Product } \\
\text { performance }\end{array}$ & $\begin{array}{l}\text { Significant relationship between Product co-development with } \\
\text { supplier and Product Innovation. Significant relationship between } \\
\text { Information sharing with supplier and Product performance. } \\
\text { Significant relationship between Product co-development with } \\
\text { customer and Product performance. Significant relationship between } \\
\text { Product Innovation and Product performance. Positive direct of } \\
\text { control variable effects of company size on Product Innovation. } \\
\text { Negative direct of control variable effects of market certainty on } \\
\text { Product Innovation. Negative direct of control variable effects of } \\
\text { market certainty on Information sharing with supplier. }\end{array}$ \\
\hline 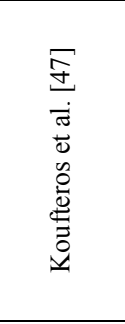 & $\begin{array}{l}\text { Concurrent } \\
\text { Engineering } \\
\text { (Internal } \\
\text { Integration) }\end{array}$ & $\begin{array}{l}\text { customer } \\
\text { integration, supplier } \\
\text { product } \\
\text { integration (black } \\
\text { box), supplier } \\
\text { process } \\
\text { integration (grey box), } \\
\text { product } \\
\text { innovation, quality, } \\
\text { profitability }\end{array}$ & $\begin{array}{c}\text { Product } \\
\text { Innovation, Quality, } \\
\text { Profitability }\end{array}$ & $\begin{array}{l}\text { Significant relationship between concurrent engineering and: } \\
\text { customer integration, black box integration, grey box integration. } \\
\text { Significant relationship between customer integration and: product } \\
\text { innovation, quality. Significant relationship between suppliers. } \\
\text { Product integration and supplier process integration. Negative } \\
\text { significant relationship between supplier product integration and } \\
\text { product innovation. Significant relationship between product } \\
\text { innovation and: quality performance, profitability. }\end{array}$ \\
\hline
\end{tabular}




\begin{tabular}{|c|c|c|c|l|}
\hline & & & \\
Cross functional & Customer and & $\begin{array}{l}\text { Significant relationship between Customer involvement in NPD and: } \\
\text { integration, NPD } \\
\text { performance }\end{array}$ & $\begin{array}{c}\text { NPD performance } \\
\text { Cross functional integration, NPD performance. Significant } \\
\text { relationship between supplier involvement in NPD and: Cross } \\
\text { functional integration, NPD performance. Significant relationship } \\
\text { between Cross functional integration and NPD performance. }\end{array}$ \\
\hline
\end{tabular}

\section{Proposed Model}

\subsection{The role of CRM in new product development}

Different definitions have been suggested for CRM in the literature. Meta Group divides CRM into three areas [50]:

- Operational CRM

- Analytical CRM

- Collaborative CRM.

\subsubsection{Operational CRM and NPD}

Operational CRM is related to automation of business processes involving contact points with the customer at the "front office". This area includes the automation of different parts in customer relationship:

- $\quad$ Enterprise Marketing Automation (EMA)

- Sales Force Automation (SFA)

- Customer Service and Support (CSS) Automation.

Operational CRM can help marketing automation through marketing campaigns. In this area, customers are provided with updated information and direct interactions with customers are facilitated. CRM systems are effective in organizing and planning these campaigns and provide appropriate channels for communications with the customers. Moreover, CRM tries to manage sales through sales force automation such as order management system and sales force support is provided. As service automation attempts at making long-term relationships with customers, it is considered a very important component. Service automation can be achieved through help desk wherein customers' requests are allocated to appropriate working forces. There has been a distinctive focus on the importance of different components of operational CRM for new product development in the literature.

Tyagi \& Sawhney [51] considered the effective role of marketing campaigns and advertisements in the NPD performance. One of important stages in NPD process is new product launch. Three factors, namely when, how and where for launching new product, directly influence the effectiveness of products in the market. Marketing strategies and plans are directly related to new product launch to market. In marketing automation, direct marketing methods can prove helpful in getting feedback from the customers, new product positioning, seeking innovative customers by effective campaigns, etc. Management of marketing campaigns can improve the market share related to new product introduction. On the contrary, poor marketing planning could lead to the failure of new products[52].

The role of the sales force in new product development has been studied by Judson et al. [53]. They found salespersons both directly and indirectly played an important role in new product development and their suggestions could be used to gather information necessary to create ideas for new products. Gordon et al.[54] stated that sales force cannot act as a reliable and valuable source for new products unless there is a structured system for receiving and collecting customers' feedback and information. Customer-related information (on new product development) collected by sales force could reside in CRM systems, in the individual files of salespersons or even their supervisors (CRM packages have such capabilities) [55].

Product support is considered an important factor in successful marketing for many products. Many aspects of support are influenced by product design; therefore, customer support requirements should be taken into account during the NPD process. Goffin and New [56] conducted an exploratory study on the relationships between customer support and service and NPD. They found that innovation managers will need to allocate enough resources to integrate customer support requirements in new product development. The study of Goffin and New [56] shows the necessity of implementing CRM and applying CSS during the NPD process to achieve new product success.

\subsubsection{Analytical CRM and new product development}

Analytical CRM involves Data Mart, Data warehouse, customer data storage, etc. which analyses customer data by using software and algorithms and presents them in a useful and available format. Analytical CRM also includes analysis of data collected through operational CRM. It bridges the gap between front office and back office. Analyses yield valuable information on market, customer behaviour and etc. In this part, market information is analysed, the successfulness of campaigns is evaluated and customer satisfaction is assessed. These analyses can be used for customer segmentation, identification of potential customers for cross-selling and up-selling, and etc. Analytical CRM can play an important role in understanding customers' needs for new products or services [57].

In addition, analytical CRM is able to determine customers' needs and predict their future needs along the path to the development of new products and services. Therefore, this element of CRM can develop the strategies of the organization in order to satisfy customer needs. Accordingly, a study by Veldhuizen et al. [39] attested to the effective role of market information processing in new product advantage and success through the new product development process. Effective information processing has been known as a challenging area because many organizations are not able to utilize market information in their NPD process [58]. These studies confirm the use of analytical CRM in organizations during the NPD process.

\subsubsection{Collaborative CRM and new product development}

Collaborative CRM includes all methods of communication (different channels) between 
organizations and customers (such as email, telephone, fax, website pages, etc.). This part supports coordination between the staff and channels. The final goal of the collaborative CRM is to make use of information gathered from different departments in order to improve the quality of customer services [59]. Interaction and integration with customers can be achieved by communicational processes which support knowledge sharing between the organization and customers [60]. In NPD, collaborative CRM supports the organization in using all kinds of the channels that facilitate contact with different customer segments to achieve the specific goals of NPD. In addition, collaborative CRM as a component of CRM provides the opportunity for the organization to get access to a larger amount of valuable customer information for new product development. Using different communicational channels for gathering customer-related information may be regarded as the input for new product development process. Therefore, collaborative CRM could provide capabilities to better NPD performance.

\subsection{SRM and new product development}

SRM as defined by the Gartner Group is the "practices needed to establish business rules, and the understanding needed for interacting with suppliers of products and services of varied criticality to the profitability of the enterprise" [61].

Other researchers have regarded SRM as a new generation of E-procurement [62], and in particular, as an integrated approach that bridges the gaps between product development, sourcing, supply and procurement planning across the value chain [63]. SRM can help the organization achieve the following competitive advantage [64]:

- Increasing satisfaction of services and purchased goods and speeding up product development through sharing suppliers knowledge and alternative technologies;

- Increasing satisfaction among suppliers;

- Lowering costs to service customers by improving business processes across the supply chain.

In order to achieve profitability, organizations must be able to choose the appropriate supplier as soon as possible, to create strategic relations and cooperate with suppliers along the lines of its goals. SRM provides the necessary processes for creating business rules to interact with suppliers and offering services related to the organization's profitability. SRM attempts to share knowledge and resources, combine competitive cores with suppliers, achieve synergy and establish a win-win relationship. SRM's advantages include decreasing costs, providing repeatable procurement, speeding up product development, attracting and retaining competitive advantage, decreasing costs for purchasing products/ services [65].

SRM can be used to evaluate suppliers' capability and select the appropriate supplier for the new product development process. Using SRM for the selection of suppliers in new product development can have advantages such as faster reaction to change the criteria of choosing suppliers - which might happen during the new product development, decreasing decision time in supplier management and the retention of valuable information in supplier management [66].

A good example for the successful implementation of SRM was in Latin America where companies utilized SRM in order to improve services level and time to market for new products (Kearney, 2004). SRM, like CRM, has capabilities which can be used in new product development. Among these capabilities are technical capabilities, support and service, purchase and marketing. All CRM solutions rely on special technologies to provide services. Similar means are available for SRM, too.

Using such tools, organizations will be able to share experiences on services and products and to lower risks related to new product development and suppliers' selection.

Like CRM which has data Mart, data warehouse and analytical instruments, suppliers' information repository is base of SRM solutions. Organizations can use the tool for segmenting suppliers in order to participate in new product development according to the new product features. The aim of the service and support section in CRM is to provide the best product with the highest quality and lowest cost. SRM pursues the same purpose within the organization in order to collect and manage information across all departments of the organization. For instance, if a problem occurs for the new product or for the quality of the product or in the production line, etc., the call management unit updates the supplier repository and the incident is dealt with. At the same time the procurement manager is informed of the incident; therefore, full-scale participation and integrated task distribution are achieved in the organization. The objective of sales force automation is to improve the efficiency of the sales force while lowering the time and costs of the sales. Meanwhile, SRM's purchase unit supports the purchase of the services and goods. This section possesses electronic catalogues, electronic RFPs and electronic online auction. SRM integrates this section with other procurement activities. Like marketing automation in CRM, the best guarantee for purchase managers to ensure their desired goods or services will be bought is to involve the suppliers in a competition. By improving marketing campaigns the organization will be able to attract a lot of suppliers. Such module allows for the selection of the suppliers according to pre-determined criteria through available channels. In this way, purchase managers can identify potential suppliers and gather the required information.

\section{Conclusion}

Several factors warrant the participation of the organization in new product development. Some of these factors include:

- Global economic stagnation motivates organizations to seek for ways to improve the efficiency of their products. Generally in the light of the knowledge gained by organizations on the supply chain, organizations are normally seeking ways to decrease the time devoted to develop the new product.

- Increasing demand for customized products such as DELL will increase complexities in new product 
development. More complex and specialized products make it impossible for organizations to go through the product development on their own.

- In order to maintain their competitive advantage in global markets organizations try to their activities and assign external resources to new technologies. In fact, in this way organizations gain considerable financial and operational benefits through the participation of their external partners.

As companies and organizations emphasize the important role of new product development in achieving competitive advantage, and as they shift from a productoriented approach to a customer-oriented one, developing mechanisms with the ability to support the approach seems necessary. On the other hand, customers' and suppliers' roles in increasing the success and decreasing the failure rate of new product development projects are undeniable. Since SRM and CRM have the capabilities to systematically facilitate the role of customers and supplies in new product development, leading to improved new product development and organizational performance, organizations are required to gain knowledge on the mechanisms through which CRM and SRM influence the performance of the new product development. Having identified the capabilities of SRM and CRM and their potential roles in NPD, this research attempted to present a model which could be helpful for organizations in their NPD activities. Future research can focus on presenting a conceptual model. This conceptual model can consider CRM resources and capabilities and SRM resources and capabilities as antecedents successful NPD in an empirical study.

\section{Acknowledgments}

The authors would like to express their appreciation to the Iranian National Science Foundation (grant number 91002765) for the financial support of this study.

\section{References}

[1] Lin, R. J.; Chen, R. H.; Chiu, K. K. S. Customer relationship management and innovation capability: an empirical study. // Industrial Management \& Data Systems. 110, 1(2010), pp.111-133.

[2] Shamsuzzoha, A.; Kyllönen, S.; Helo, P. Collaborative customized product development framework. // Industrial Management \& Data Systems. 109, 5(2009), pp.718-735.

[3] Chou, C.; Yang, K. P. The interaction effect of strategic orientations on new product performance in the high-tech industry: A nonlinear model. // Technological Forecasting and Social Change. 78, 1(2011), pp. 63-74.

[4] Hsieh, M. H.; Tsai, K. H.; Wang, J. R. The moderating effects of market orientation and launch proficiency on the product advantage-performance relationship. // Industrial Marketing Management. 37, 5(2008), pp. 580-592.

[5] Rauniar, R.; Doll, W.; Rawski, G.; Hong, P. Shared knowledge and product design glitches in integrated product development. // International Journal of Production Economics. 114, 2(2008), pp. 723-736.

[6] Sun, H.; Yau, H. K.; Ming Suen, E. K. The Simultaneous Impact of Supplier and Customer Involvement on New Product Performance. // Journal of Technology Management \& Innovation. 5, 4(2010), pp.70-82.
[7] Chien, S. H.; Chen, J. Supplier involvement and customer involvement effect on new product development success in the financial service industry. // The Service Industries journal. 30, 2(2010), pp.185-201.

[8] Ramani, G.; Kumar, V. Interaction orientation and firm performance. // Journal of Marketing. 72, 1(2008), pp. 2745.

[9] Ernst, H.; Hoyer, W. D.; Krafft, M.; Krieger, K. Customer relationship management and company performance-the mediating role of new product performance. // Journal of the Academy of Marketing Science. 39, 2(2011), pp. 290306.

[10] Sahay, B. S.; Ranjan, J. Real time business intelligence in supply chain analytics. // Information Management \& Computer Security. 16, 1(2008), pp. 28-48.

[11] Reinartz, W.; Krafft, M.; Hoyer, W. D. The customer relationship management process: Its measurement and impact on performance. // Journal of marketing research. 41, 3(2004), pp. 293-305.

[12] Herrmann, J. W.; Hodgson, B. SRM: leveraging the supply base for competitive advantage. // Proceedings of the SMTA International Conference, Chicago, IL (Vol. 1). (2001).

[13] Park, J.; Shin, K.; Chang, T. W.; Park, J. An integrative framework for supplier relationship management. // Industrial Management \& Data Systems. 110, 4(2010), pp. 495-515.

[14] Cooper, L. P. A research agenda to reduce risk in new product development through knowledge management: a practitioner perspective. // Journal of Engineering and Technology Management. 20, 1-2(2003), pp. 117-140.

[15] Edgett, S.; Shipley, D.; Forbes, G. Japanese and British companies compared: contributing factors to success and failure in NPD. // Journal of Product Innovation Management. 9, 1(1992), pp. 3-10.

[16] Griffin, A.; Belliveau, P.; Association, P. D.; M. Drivers of NPD success: The 1997 PDMA report. PDMA. (1997).

[17] Palacios, T. M. B.; González, F. J. M. Assessing the validity of new product development techniques in Spanish firms. // European Journal of Innovation Management. 5, 2(2002), pp. 98-106.

[18] Tennant, C.; Roberts, P. The creation and application of a self-assessment process for new product introduction. // International journal of project management. 21, 2(2003), pp. 77-87.

[19] Lettice, F., Roth, N., \&Forstenlechner, I. Measuring knowledge in the new product development process. //International Journal of Productivity and Performance Management, 55, 3/4(2006), pp.217-241.

[20] Murphy, S. A.; Kumar, V. The front end of new product development: a Canadian survey. // R\&D Management. 27, 1(1997), pp. 5-15.

[21] Rogers, H.; Ghauri, P.; Pawar, K. S. Measuring international NPD projects: an evaluation process. // Journal of Business \& Industrial Marketing. 20, 2(2005), pp. 79-87.

[22] Nambisan, S. Designing virtual customer environments for new product development: Toward a theory. // Academy of Management Review. 27, 3(2002), pp. 392-413.

[23] Performance measurement Group LLC (a PRTM company). Co-Development survey results, January PDMA/MRT Co-Development conference. (2002).

[24] Wagner, S. M. Intensity and managerial scope of supplier integration. // Journal of Supply Chain Management. 39, 4(2003), pp. 4-15.

[25] Van Echtelt, F. E. A.; Wynstra, F.; Van Weele, A. J.; Duyesters, G. Managing supplier involvement in NPD: a multiple-case study. // Journal of Product Innovation Management. 25, (2008), pp. 180-201. 
[26] Petersen, K. J.; Handfield, R. B.; Ragatz, G. L. A Model of Supplier Integration into New Product Development. // Journal of Product Innovation Management. 20, 4(2003), pp. 284-299.

[27] Ragatz, G. L.; Handfield, R. B.; Petersen, K. J. Benefits associated with supplier integration into new product development under conditions of technology uncertainty. // Journal of Business Research. 55, 5(2002), pp. 389-400.

[28] Johnsen, T. E. Supply network delegation and intervention strategies during supplier involvement in new product development. // International Journal of Operations \& Production Management. 31, 6(2011), pp. 686-708.

[29] McIvor, R.; Humphreys, P.; Cadden, T. Supplier involvement in product development in the electronics industry: a case study. // Journal of Engineering and Technology Management. 23, 4(2006), pp. 374-397.

[30] Primo, M. A. M.; Amundson, S. D. An exploratory study of the effects of supplier relationships on new product development outcomes. // Journal of Operations Management. 20, 1(2002), pp. 33-52.

[31] Von Corswant, F.; Tunalv, C. Coordinating customers and proactive suppliers: A case study of supplier collaboration in product development. // Journal of Engineering and Technology Management. 19, 3-4(2002), pp. 249-261.

[32] Littler, D.; Leverick, F.; Wilson, D. Collaboration in new technology based product markets. // International journal of technology management. 15, 1(1998), pp. 139-159.

[33] Hartley, J. L.; Zirger, B. J.; Kamath, R. R. Managing the buyer-supplier interface for on-time performance in product development. // Journal of Operations Management. 15, 1(1997), pp. 57-70.

[34] Feng, T.; Sun, L.; Zhu, C.; Sohal, A. S. Customer orientation for decreasing time-to-market of new products: IT implementation as a complementary asset. // Industrial Marketing Management. 41, 6(2012), pp. 929-939.

[35] Bonner, J. M. Customer interactivity and new product performance: Moderating effects of product newness and product embeddedness. // Industrial Marketing Management. 39, 3(2010), pp. 485-492.

[36] Carbonell, P.; Rodríguez-Escudero, A. I.; Pujari, D. Customer Involvement in New Service Development: An Examination of Antecedents and Outcomes. // Journal of Product Innovation Management. 26, 5(2009), pp. 536-550.

[37] Wei, Y. S.; Atuahene-Gima, K. The moderating role of reward systems in the relationship between market orientation and new product performance in China. // International Journal of Research in Marketing. 26, 2(2009), pp. 89-96.

[38] Swink, M.; Song, M. Effects of marketing-manufacturing integration on new product development time and competitive advantage. // Journal of Operations Management. 25, 1(2007), pp. 203-217.

[39] Veldhuizen, E.; Hultink, E. J.; Griffin, A. Modeling market information processing in new product development: An empirical analysis. // Journal of Engineering and Technology Management. 23, 4(2006), pp. 353-373.

[40] Langerak, F.; Hultink, E. J.; Robben, H. S. J. The impact of market orientation, product advantage, and launch proficiency on new product performance and organizational performance. // Journal of Product Innovation Management. 21, 2(2004), pp. 79-94.

[41] Thomas, E. Supplier integration in new product development: Computer mediated communication, knowledge exchange and buyer performance. // Industrial Marketing Management, 42, 6(2013), pp. 890-899.

[42] Parker, D. B.; Zsidisin, G. A.; Ragatz, G. L. Timing and extent of supplier integration in new product development: a contingency approach. // Journal of Supply Chain Management. 44, 1(2008), pp. 71-83.
[43] Koufteros, X. A.; Edwin Cheng, T. C.; Lai, K. H. "Blackbox" and "gray-box" supplier integration in product development: Antecedents, consequences and the moderating role of firm size. // Journal of Operations Management. 25, 4(2007), pp. 847-870.

[44] Petersen, K. J.; Handfield, R. B.; Ragatz, G. L. Supplier integration into new product development: coordinating product, process and supply chain design. // Journal of Operations Management. 23, 3-4(2005), pp. 371-388.

[45] Chung, S. A.; Kim, G. M. Performance effects of partnership between manufacturers and suppliers for new product development: the supplier's standpoint. // Research Policy. 32, 4(2003), pp. 587-603.

[46] Walter, A. Relationship-specific factors influencing supplier involvement in customer new product development. // Journal of Business Research. 56, 9(2003), pp. 721-733.

[47] Koufteros, X.; Vonderembse, M.; Jayaram, J. Internal and external integration for product development: the contingency effects of uncertainty, equivocality, and platform strategy. // Decision Sciences. 36, 1(2005), pp. 97133.

[48] Lau, A. K. W.; Tang, E.; Yam, R. Effects of Supplier and Customer Integration on Product Innovation and Performance: Empirical Evidence in Hong Kong Manufacturers. // Journal of Product Innovation Management. 27, 5(2010), pp. 761-777.

[49] Feng, T.; Wang, D. Supply chain involvement for better product development performance. // Industrial Management \& Data Systems. 113, 2(2013), pp. 190-206.

[50] Greenberg, P. CRM at the Speed of Light: Social CRM Strategies, Tools, and Techniques for Engaging Your Customers. McGraw-Hill. (2010).

[51] Tyagi, R. K.; Sawhney, M. S. High-Performance Product Management: The Impact of Structure, Process, Competencies, and Role Definition. // Journal of Product Innovation Management. 27, 1(2010), pp. 83-96.

[52] Chan, S. L.; Ip, W. H. A dynamic decision support system to predict the value of customer for new product development. // Decision Support Systems. 52, 1(2011), pp. 178-188.

[53] Judson, K.; Schoenbachler, D. D.; Gordon, G. L.; Ridnour, R. E.; Weilbaker, D. C. The new product development process: let the voice of the salesperson be heard. // Journal of Product \& Brand Management. 15, 3(2006), pp. 194202.

[54] Gordon, G. L.; Schoenbachler, D. D.; Kaminski, P. F.; Brouchous, K. A. New product development: using the salesforce to identify opportunities. // Journal of Business \& Industrial Marketing. 12, 1(1997), pp. 33-50.

[55] Zahay, D.; Griffin, A.; Fredericks, E. Sources, uses, and forms of data in the new product development process. // Industrial Marketing Management. 33, 7(2004), pp. 657666.

[56] Goffin, K.; New, C. Customer support and new product development-An exploratory study. // International Journal of Operations \& Production Management. 21, 3(2001), pp. 275-301.

[57] Bae, S. M.; Ha, S. H.; Park, S. C. A web-based system for analyzing the voices of call center customers in the service industry. //Expert Systems with Applications. 28, 1(2005), pp. 29-41.

[58] Cooper, R. G.; Edgett, S. J. Best Practices in Product Innovation: What Distinguishes Top Performance? Product Development Institute Inc., Ancaster, 2003.

[59] Edwards, J. Get It Together with Collaborative CRM. Inside CRM. Tippit, 2009.

[60] Gales, L.; Mansour-Cole, D. User involvement in innovation projects: Toward an information processing 
model. // Journal of Engineering and Technology Management. 12, 1-2(1995), pp. 77-109.

[61] Hope-Ross D.; Spencer C. SRM is not yet a suite-spot, Gartner Group, Oct. 2001.

[62] Segev A.; Gebauer J.; Färber F. The Market for Internetbased Procurement Systems, Haas School of Business, University of California, Feb, 2000.

[63] I2 Inc. www.i2.com, (2001).

[64] Lang, A.; Paravicini, D.; Pigneur, Y.; Revaz, E. From Customer Relationship Management (CRM) to Supplier Relationship Management (SRM). HEC Lausanne2002. Available: http://www.hec.unil.ch/yp/Pub/02-SRM.pdf, (2002).

[65] Pigneur, Y. From Customer Relationship Management to Supplier Relationship Management, http://www.hec.unil.ch/yp/Pub/02-SRM.PDF. (2004).

[66] Lee, W. B.; Cheung, C. F.; Lau, H. C. W.; Choy, K. L. Development of a Web-based enterprise collaborative platform for networked enterprises. // Business Process Management Journal. 9, 1(2003), pp. 46-59.

\section{Authors' addresses}

Salman Nazari-Shirkouhi, corresponding author

School of Industrial Engineering, College of Engineering,

University of Tehran, Tehran, Iran

E-mail: snnazari@ut.ac.ir

Abbas Keramati

School of Industrial Engineering, College of Engineering,

University of Tehran, Tehran, Iran

\section{Kamran Rezaie}

School of Industrial Engineering, College of Engineering,

University of Tehran, Tehran, Iran 